\title{
A new model of selective photothermolysis to aid laser treatment of port wine stains
}

\author{
LI Dong $^{1}$, HE YaLing $^{1 *}$, WANG GuoXiang ${ }^{1,2 *}$, WANG YongXian ${ }^{3} \&$ YING ZhaoXia $^{3}$ \\ ${ }^{1}$ State Key Laboratory of Multiphase Flow in Power Engineering, Xi'an Jiaotong University, Xi'an 710049, China; \\ ${ }^{2}$ Department of Mechanical Engineering, The University of Akron, Akron 44325-3903, USA, \\ ${ }^{3}$ Department of Dermatology, Medical School of Xi'an Jiaotong University, Xi'an 710049, China
}

Received March 29, 2012; accepted July 2, 2012

\begin{abstract}
Pulsed dye lasers equipped with cryogen spray cooling (CSC) are now widely used to treat vascular malformation such as port wine stains (PWSs). This paper presents a new integrated model that can quantitatively simulate the cooling of the skin and the heating of the targeted blood vessels in PWSs during laser treatment. The new model is based on the classical homogeneous multi-layer skin model that treats PWS-containing dermis as a mixture of dermal tissue and homogeneously distributed blood. Light propagation in skin and PWSs is simulated by a Monte-Carlo method, which provides accurate description of the light scattering and absorption in the skin. Thermal response of a targeted vessel in the new model is then obtained from the thermal analysis of a Krogh-unit that consists of the vessel and the surrounding dermal tissues and is buried in PWSs. The results from the multi-layer skin model provide appropriate laser influence input as well as the initial thermal condition for the micro-model of the Krogh-unit. A general dynamic relation is also introduced on the surface of skin to quantify the convective cooling of CSC. The model is then applied to dye-laser treatment (wavelength of $585 \mathrm{~nm}$ ) of PWSs with CSC. Numerical results demonstrate that the present model is able to quantify thermal response of a deeply buried blood vessel in PWSs as a discrete blood vessel does, with a more realistically estimate of the sheltering effect of the dermal tissue (scattering) and blood vessels (absorption) in front of the targeted vessel. To understand the poor response of PWSs in clinic, the thermal characteristics of a targeted vessel was simulated under various conditions. The effects of two morphological parameters, the vessel diameter and the burying depth of the vessel, are then systematically investigated under various pulse durations and fluences of laser. A threshold fluence for given vessel diameter and depth is then estimated quantitatively under the condition of the optimal laser pulse duration. The results indicate that significant high threshold fluence is needed for large vessels buried deeply in the dermis, explaining the physics of the difficulty in clinic of complete clearance of PWSs. The results provide guidance to the clinic selection of the laser pulse duration and laser energy fluence for given PWSs.
\end{abstract}

cryogen spray cooling, laser treatment of PWS, poor responding lesions, vessel depth, vessel diameter

Citation: $\quad \mathrm{Li} \mathrm{D}, \mathrm{He}$ Y L, Wang G X, et al. A new model of selective photothermolysis to aid laser treatment of port wine stains. Chin Sci Bull, 2013, 58: 416-426, doi: $10.1007 / \mathrm{s} 11434-012-5444-0$

Port wine stain (PWS) birthmark is a congenital and progressive vascular malformation of the capillaries in dermis, which occurs in approximate $0.3 \%$ of new born children [1]. Treatment of port wine stains is based on the principle of selective photothermolysis brought forward by Anderson and Parrish [2]. Based on their theory, light (laser) is preferably absorbed by hemoglobin in PWS blood vessels,

*Corresponding authors (email: yalinghe@mail.xjtu.edu.cn; gwang@uakron.edu) causing irreversible thermal damage, thrombosis, and eventually, permanent photocoagulation in the PWS vessels. Unfortunately, a significant amount of energy in laser beam is absorbed by melanin in the basal layer of the epidermis, leading to skin dyspigmentation or hypertrophic scarring [3]. Skin cooling techniques such as cryogen spray cooling (CSC) was then introduced to selectively cool the epidermis prior to laser irradiation to allow higher energy used in clinic [3-6]. Nowadays, pulsed dye lasers (PDL) of wavelengths 
from 585 to $600 \mathrm{~nm}$ together with CSC become the treatment of choice for PWSs. A complete clearance of PWSs is, however, rare (less than 25\%) with many PWSs respond poorly to laser treatment [7-9].

Numerical method is an effective tool that has been used widely in the literature to investigate the laser treatment of PWSs for better understandings of the mechanism of thermal response in biological tissues, quantification of the thermal damage, optimization of surgery parameters, and development of new treatment protocols [10]. Modeling laser PWS requires accurate prediction of light propagation in human tissues, the energy deposition and corresponding temperature distribution in skin containing PWS. An accurate description of the dynamic cooling effect of cryogen spray prior to laser irradiation is also important in quantifying the laser treatment of PWSs.

A convective thermal boundary condition is usually used to quantify cryogen spray cooling in laser PWS modeling. Due to the lack of the experimental data, the early models of laser PWS usually employed a constant heat transfer coefficient $(h)$ during the short-pulsed cooling process [11-14]. Recent experiments of cryogen spray cooling, however, reveal a strong dynamic behavior in cryogen spray cooling $[15,16]$. Aguilar et al. [17] have introduced a time-dependent $h$ in their simulation by assuming a linear variation of $h$ with time. Jia et al. [18] have even used the measured heat flux from their experiments in their model. Recently, based on the experimental data, the present authors have introduced a universal relation of dynamic varying heat transfer coefficient during cryogen spray $[19,20]$. This relation includes both the spatial and temporal variation of $h$ during the short-pulsed spray cooling process.

The Monte-Carlo method has found a wide use in modeling and quantifying the light propagation and energy deposition in human skin during laser PWSs [10,21-25]. The method offers a flexible approach to track the photon transport in biological tissues. Due to the complex structure of multi-component human skin tissues, simplified models of a multi-layer skin are usually used in Monte-Carlo calculation of laser PWS. The skin models usually include an epidermal layer with chromophore melanin uniformly distributed throughout the layer. Various models of laser PWS in the literature lie in the difference in the ways of treating blood vessels or the distribution of the hemoglobin in the blood vessel. The discrete vessel models [18,21,22] treat pathological blood vessels in PWSs as finite discrete cylindrical tubes parallel to each other and regularly distributed in dermis. On the other hand, the homogeneous medium models [23-25] treat the PWS as a special tissue layer embedded within the dermis. Within the PWS layer, the hemoglobin (blood) is assumed to be homogeneously mixed with the dermal tissues. Both kinds of models have been used in the literature to investigate the laser-tissue interaction during laser PWS. The discrete vessel model provides a direct visualization of laser-vessel interaction during surgery, while the homogeneous model is more computational efficient, and to certain degree closer to the real PWS structure.

This paper presents a new mathematical model for simulation of laser treatment of PWSs with CSC. The model is based on the classical homogeneous-medium, multi-layer skin model of PWSs, and treats the PWS-containing dermis as a homogeneous mixture of dermal tissue and uniformly distributed blood. The multi-layer Monte-Carlo method is then used to calculate the light propagation in the skin layers including the melanin-containing epidermis and PWS-containing dermis. It was demonstrated in the literature that such a multi-layer Monte-Carlo method can accurately simulate the light scattering of dermal tissues and light absorption of blood vessels in PWSs [10]. The model is therefore able to provide an adequate description of the sheltering effect of melanin in epidermis and the blood vessels in front of the targeted vessels. A Krogh-unit is then introduced locally in PWSs to represent a targeted blood vessel. The Krogh-unit consists of the targeted vessel and the surrounding tissues. Thermal analysis of such a Kroghunit under the macro-thermal environment at the given location provides an accurate description of the thermal response of the targeted blood vessels for given clinic conditions. Numerical results are presented to illustrate the basic characteristics of thermal response of a targeted blood vessel in laser surgery under typical clinic conditions. A parametric study is then performed to investigate the effect of clinic parameters such as laser energy and spurt duration of cryogen spray. The effect of vessel morphological parameters such as the diameter of the blood vessels and the depth of the blood vessel is also studied. Threshold laser fluences for thermal vascular injury of vessels are estimated under various conditions.

\section{Physical model and mathematical description}

The schematic of the new mathematical model is shown in Figure 1, including a two-layer skin model and a micro-scale Krogh-unit located within PWSs. The macroscopic skin model consists of an epidermal layer and a bloody dermal layer that represents blood vessels of PWSs. The epidermal layer contains melanin that is strongly light-absorbing and is assumed to be uniformly distributed in the layer. A uniformly-distributed blood of a given volumetric percentage is used here to simplify the treatment of blood vessels in the PWS layer, as did by many researchers in the literature [23-25]. Such a treatment of PWSs with the blood homogeneously distributed in dermis makes it simple for calculation of light propagation and energy deposition by the Monte-Carlo method. As demonstrated by Verkruysse et al. [25], such a treatment could provide a quantitative prediction of the light competing effect between numerous blood vessels and the sheltering effect from shallower vessels. The difference of the present model from any of early models in 


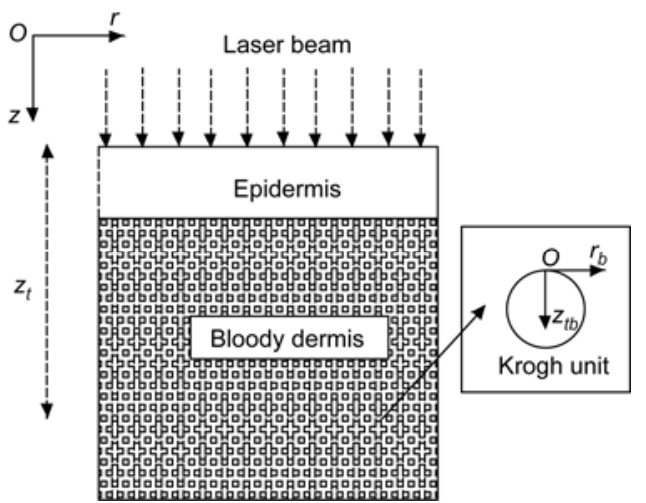

Figure 1 A schematic of the macroscopic skin model and the microscopic Krogh-unit.

the literature lies in the treatment of thermal response of the skin tissue and the targeted blood vessels in the PWS layer. A macroscopic thermal model as the classical homogeneous model is first employed to simulate the dynamic change of the temperatures of both epidermis and dermis with blood. The macroscopic thermal model does include, however, a dynamic varying convective heat transfer coefficient on skin surface during the short spurt of cryogen spray. The macroscopic model provides a thermal environment for the thermal analysis of the targeted blood vessel within a classical Krogh unit [26] that consists of the targeted vessel and the surrounding tissues (see Figure 1). The Krogh-unit provides an accurate description of a discrete blood vessel during laser treatment. The input light fluence on the top of the blood vessel in the Krogh-unit comes from the Monte-Carlo calculation of the macro-model. The attenuation of laser light within the blood in the vessel, governed by the Lambert-Beer law [25], is also included. The present micromodel of the Krogh-unit introduces the effect of the morphological configurations (e.g. the depth and the diameter) of the targeted vessel. Such an integrated treatment avoids the morphological complexity of the PWSs while provides an accurate description of the thermal response of individual blood vessels during laser treatment.

\subsection{Light distribution and heat transfer in epidermis and bloody dermis: the Macro-model}

The skin is assumed to have two parallel layers including an epidermal layer and a bloody dermal layer. The melanin (5\% volumetric percentage [18]) and blood (10\% volumetric percentage [27]) are assumed to be homogeneously distributed in two layers, respectively.

The heat conduction equation in epidermis and bloody dermis can be written as follows:

$$
\rho_{i} c_{p i} \frac{\partial T\left(r, z_{t}, t\right)}{\partial t}=\lambda_{i} \nabla^{2} T\left(r, z_{t}, t\right)+Q_{i}\left(r, z_{t}\right) \quad i=e, b d,
$$

where, $\rho$ is the density, $c_{p}$ the specific heat, $\lambda$ the thermal conductivity, $T$ the temperature, and $t$ the time. $Q_{i}$ is the source of volumetric heat generation due to the absorption of laser light in each layer and can be determined as follows:

$$
Q_{i}\left(r, z_{t}\right)=\mu_{a i} \Phi\left(r, z_{t}\right) \quad i=e, b d,
$$

where $\Phi\left(r, z_{t}\right)$ is the light fluence at the radial position $r$ (measured from the center of the beam) and the skin depth $z_{t}$ (measured from the skin surface) and is calculated by the multi-layer Monte Carlo method [28]. Subscripts $e$ and $b d$ stand for epidermal layer and bloody dermal layer, respectively. Due to a short time period involved in CSC and pulsed laser irradiation, the blood perfusion effect is safely neglected [29,30].

On the skin surface the standard convection condition is used to describe the cooling effect of CSC:

$$
\begin{aligned}
& -\left.\lambda_{e} \frac{\partial T}{\partial z}\right|_{z_{t}=0} \\
& =\left\{\begin{array}{l}
h_{c}(t)\left[T(r, 0, t)-T_{c}\right], \text { during CSC, } \\
h_{a}\left[T(r, 0, t)-T_{a}\right], \text { during and after laser heating, }
\end{array}\right.
\end{aligned}
$$

where $T_{a}$ is the air temperature which is set to be $25^{\circ} \mathrm{C} ; T_{c}$ is the cryogen film temperature, which is calculated form single droplet evaporation model [31]. The convection heat transfer coefficients, $h_{a}$, represents normal cooling after CSC. The convective heat transfer coefficient, $h_{c}$, during $\mathrm{CSC}$, is evaluated from the following generalized correlation $[19,20]$ :

$$
h_{o}^{*}(\tau)= \begin{cases}\tau, & \tau \leqslant 1.0, \\ 1.0-0.35(\tau-1), & 1.0<\tau \leqslant 3.0, \\ 0.3-0.02(\tau-3), & 3.0<\tau \leqslant 8.0, \\ 0.2-0.0125(\tau-8), & 8.0<\tau,\end{cases}
$$

where $h^{*}(\tau)=h_{c} / h_{o, \text { max }}$ is the normalized convection heat transfer coefficient, $\tau=t / t_{\max }$ the non-dimensional time, $h_{o, \max }$ is the maximum value of $h_{c}$ during spray; and $t_{\max }$ is the time at which $h_{o, \max }$ occurs. It is noticed that above relation includes two free parameters, i.e., $t_{\max }$ and $h_{o \text {, max }}$, which have to be experimentally determined and are available only for R134a [19,20]. In this paper, we assume that the relation is applicable to other cryogens like R407c and R404a, as suggested from our recent experiments [32]. But $h_{o, \max }$ is treated in the model as a free parameter and estimated for a given CSC condition so that the skin surface temperature is stabilized at the corresponding boiling point of the given cryogen after liquid cryogen pool is formed on the skin surface [15].

\subsection{Light distribution and laser heating in a target blood vessel: the Micro-model}

The microscopic thermal model includes a Krogh-unit that 
is composed of a vascular compartment (the blood vessel) and the non-vascular (dermis) compartment [26]. Within such a Krogh-unit, we consider both light absorption within the blood vessel and heat conduction in the blood and the surrounding tissues. The dimension of the Krogh-unit is determined by the blood vessel diameter and the blood volume fraction (assumed to be $10 \%$ in this paper [27]).

With respect to the coordinate system as shown in Figure 1 , the energy equation for the Krogh unit at location $\left(r, z_{t}\right)$ can be written as follows:

$\rho_{i} c_{p i} \frac{\partial T\left(r_{b}, z_{t b}, t\right)}{\partial t}=\lambda_{i} \nabla^{2} T\left(r_{b}, z_{t b}, t\right)+Q_{i}\left(r_{b}, z_{t b}\right), \quad i=d, b$,

where subscript $i$ represents either dermal tissue $(d)$ or blood $(b) . Q_{i}$ is the source of volumetric heat generation due to the absorption of laser light in either blood or dermal tissue. For the wavelength (e.g. $585 \mathrm{~nm}$ ) of the clinic lasers, the energy absorption by dermal tissue can be neglected and the energy absorption within the targeted blood vessel can be calculated in the following way:

$$
Q_{b}\left(r_{b}, z_{t b}\right)=\mu_{a} \Phi\left(r_{b}, z_{t b}\right),
$$

where $\mu_{a}$ is the absorption coefficient of blood and $\Phi\left(r_{b}, z_{t b}\right)$ is the light fluence within the targeted blood vessel within the Krogh unit at radial position $r_{b}$ in depth of $z_{t b}$ (measured from the top of the vessel). As the reduced scattering coefficient of the blood is much smaller than the absorption coefficient at the wavelengths of clinic interested, the attenuation of the light fluence within the target blood vessel can be calculated based on the Lambert-Beer's law [25]:

$$
\Phi\left(r_{b}, z_{t b}\right)=\Phi\left(r, z_{t}\right) \exp \left[-\mu_{a} z_{t b}\right],
$$

where $\Phi\left(r, z_{t}\right)$ is the input light fluence at the top of the blood vessel and is calculated from the multi-layer Monte Carlo method in our Macro-model.

Since we are primarily interested in the heating of the blood vessel within the Krogh unit, we confine our thermal analysis of the Krogh unit to the short time period during and immediate after laser irradiation. During such a short time period, there should be only limited thermal diffusion into the dermis from the blood vessel within the Krogh unit.
Therefore, we can confine our Krogh unit to a small volume with the outer boundary of the Krogh unit remaining at the initial skin temperature before laser irradiation (This temperature may be lower than the normal skin temperature due to cryogen spray cooling before laser irradiation). Once the light influence, $\Phi\left(r, z_{t}\right)$, within the bloody PWS layer is estimated from the macro-model through the multi-layer Monte-Carlo method, the thermal response of the targeted blood vessel can be calculated from the present micromodel of a Krogh unit. In cases when the pulse duration is long and the thermal boundary layer in dermal tissue reaches the outer boundary of the Krogh-unit, a symmetric, zero flux condition will be used instead, assuming the Kroghunit repeats itself within PWSs.

An in-house computer code was developed based on the Matlab platform. Detailed description of the numerical methods used, including the Monte-Carlo method, and benchmarks of the numerical code can be found in [33]. In this study, we choose a laser of wavelength of $585 \mathrm{~nm}$, typical for modern pulsed dye-lasers. The corresponding thermal and optical properties of epidermis, dermis, and blood are given in Table $1[11,34]$. Table 1 also provides the bulk optical and thermal properties of bloody dermis for $p \%$ blood volumetric percentage. The laser diameter of a flat-hat beam profile is chosen as $5 \mathrm{~mm}$. The thickness of the epidermal layer is $50 \mu \mathrm{m}$ and the thickness of the bloodcontaining dermal layer is $550 \mu \mathrm{m}$. The initial skin temperature is assumed to be at $30^{\circ} \mathrm{C}$ [11]. The blood vessels are assumed to be irreversibly damaged at the average temperature of $100^{\circ} \mathrm{C}$ [35], the corresponding laser fluence is then called threshold energy fluence for thermal vascular injury of the targeted vessel.

\section{Results and discussion}

An accurate description of the early cryogen spray cooling is prerequisite for any models to quantitatively simulate the laser treatment of PWSs. Therefore, the first part of the paper will focus on the treatment of CSC in the model and to demonstrate the soundness of the present model for cooling of the skin during spray before laser irradiation. In this case,

Table 1 Thermal and Optical properties of skin components [11,34]

\begin{tabular}{lcccc}
\hline \multicolumn{1}{c}{ Properties } & Epidermis & Dermis & Blood & Bloody dermis with $p \%$ blood volumetric percentge \\
\hline Density $\rho\left(\mathrm{kg} / \mathrm{m}^{3}\right)$ & 1120 & 1090 & 1060 & $\rho_{b \times} p \%+\rho_{d \times}(1-p \%)$ \\
Thermal conductivity $\lambda\left(\mathrm{kW} \mathrm{m}^{-1} \mathrm{~K}^{-1}\right)$ & 0.34 & 0.41 & 0.55 & $\lambda_{b \times} p \%+\lambda_{d \times}(1-p \%)$ \\
Specific heat $c\left(\mathrm{~J} \mathrm{~kg}^{-1} \mathrm{~K}^{-1}\right)$ & 3200 & 3500 & 3600 & $C_{b \times} \%+C_{d \times}(1-p \%)$ \\
Absorption coefficient $\mu_{a}\left(\mathrm{~cm}^{-1}\right)$ & 20 & 2.4 & 191 & $\mu_{a b \times} \%+\mu_{a d \times}(1-p \%)$ \\
Scattering coefficient $\mu_{s}\left(\mathrm{~cm}^{-1}\right)$ & 470 & 129 & 0.99 & $\mu_{s b \times} p \%+\mu_{s d \times}(1-p \%)$ \\
Anisotropy index $g$ & 0.79 & 0.79 & 1.33 & $g_{b \times} p \%+g_{d \times}(1-p \%)$ \\
Refractive index $n$ & 1.37 & 1.37 & $n_{b \times} p \%+n_{d \times}(1-p \%)$ &
\end{tabular}


only the thermal part of the macroscopic thermal model will be activated. We will examine in detail the dynamic cooling of epidermal layer and blood-contained dermal layer under various cooling conditions, controlled by the spurt duration of the spray and the cryogen boiling temperature. The spurt durations investigated ranges from 10 to $200 \mathrm{~ms}$. Three kinds of cryogens are considered with the boiling points of $-26 \mathrm{C}$ for R134a, $-43^{\circ} \mathrm{C}$ for R $407 \mathrm{c}$ and $-48^{\circ} \mathrm{C}$ for $\mathrm{R} 404 \mathrm{a}$, respectively. Once having demonstrated the validity of the CSC model, we focus on the thermal response of the targeted blood vessels buried in dermis by using the microscopic model of the Krogh-unit. The initial blood and dermal temperature within the Krogh-unit as well as the laser fluence input in the micro-model, however, are coming from the macroscopic thermal model that includes both skin cooling due to CSC before laser irradiation and the MonteCarlo simulation of laser propagation within the skin. Two major morphological parameters of the blood vessel, i.e., the diameter of the vessel $(d)$ and the depth $\left(z_{t}\right)$ from skin surface, will be investigated by examining the variation of the mean temperature of the targeted blood vessel during laser surgery. In particular, we examine the effect of these morphological parameters on the energy threshold for thermal injury of the blood vessels. Finally, a map of threshold laser fluence for thermal vascular injury will be created for typical size and depth of the blood vessels in PWSs.

\subsection{Dynamic variation of skin temperature during CSC}

Figure 2 shows the distributions of the centerline skin temperature $(r=0)$ within the skin at the end of spray for five spurt durations: 10, 30, 60, 100, and $200 \mathrm{~ms}$ with cryogen R134a. As one found, a significant drop of the epidermis temperature can be obtained during spray for all spurt durations. The lowest surface temperature may reach about $-32^{\circ} \mathrm{C}$ which will not change once the spurt duration is longer than $30 \mathrm{~ms}$. Although the skin surface temperature drops low but the entire dermal layer remains almost at the same initial skin temperature. This is because of a fairly poor thermal conductivity of the human tissue. Such a selective cooling of cryogen spray is desired for laser treatment of vascular vessels [36]. The CSC provides the protection of melanin-containing epidermis by lowering its temperature before laser irradiation while keeps the temperature of inner vascular vessels untouched. Figure 2 indicates that the spurt duration has a strong effect on such selective cooling. For example, a spurt duration of $30 \mathrm{~ms}$ may be sufficient for the surface temperature to be reduced to its minimum value, but the deep epidermis (e.g. $z_{t}=50 \mu \mathrm{m}$ ) is still at a fairly high temperature $\left(19.7^{\circ} \mathrm{C}\right)$ and would not be protected. A longer spurt duration than $30 \mathrm{~ms}$ for R134a is therefore needed. On the other hand, the spurt duration should be controlled to cool the epidermis only. An excessive long spurt may spread the cooling effect into the dermis

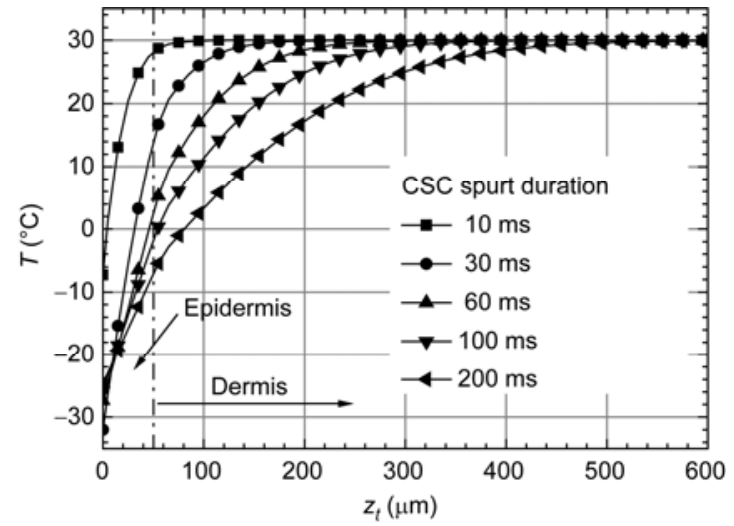

Figure 2 Centerline temperature distributions along the tissue depth for five spurt durations: 10, 30, 60, 100 and $200 \mathrm{~ms}$ ( $r=0$, cryogen: R134a).

instead of further cooling down the epidermis, as one can see for the cases when the spurt durations were longer than $100 \mathrm{~ms}$. In the case of an extremely long spurt of $200 \mathrm{~ms}$, the cooling effect penetrated deep into the bloody dermis (more than $400 \mu \mathrm{m}$ deep), undesired in laser surgery.

Figure 3 shows the dynamic variations of the temperature at four centerline locations: $z_{t}=0$ (i.e., the skin surface), 50 (at the interface between the epidermis and dermis), 100 and $300 \mu \mathrm{m}$. The spurt duration is $100 \mathrm{~ms}$ with R134a as cryogen. Again, the results show a strong selective cooling with the entire epidermal layer cooled down below the freezing point at the end of spray, while the bloody dermis remained at a high temperature. Figure 3 shows an interesting variation of the skin surface temperature during cryogen spray, i.e., the surface temperature first decreases to a minimum value (about $-32^{\circ} \mathrm{C}$ ) before it gradually approaches a temperature stabilized at the boiling point of the cryogen R134a. Such a dynamic variation of the surface temperature agrees well with the experimental measurements $[15,16]$, demonstrating the validity of the present treatment of convective heat transfer of spray cooling. During CSC, the liquid droplets reduce their temperatures to that much lower than the

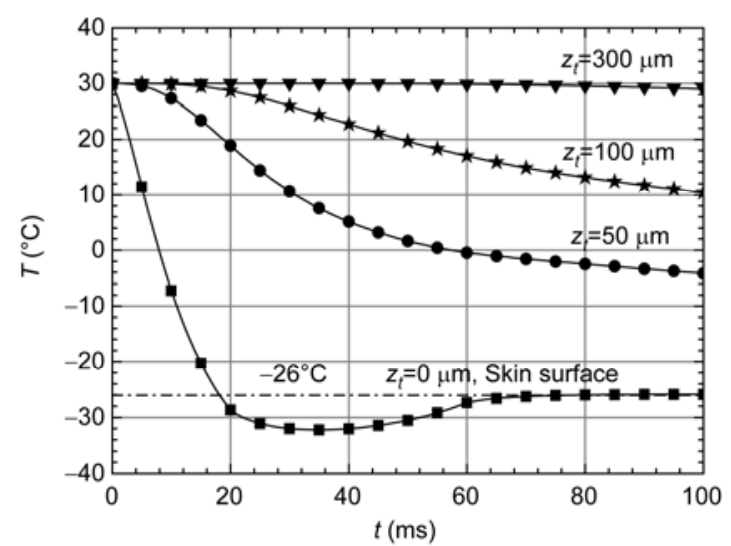

Figure 3 Dynamic variation of four centerline temperatures with time at four depths: $z_{t}=0,50,100$ and $300 \mu \mathrm{m}$ ( $r=0, \mathrm{R} 134 \mathrm{a}$, spurt duration: $\left.100 \mathrm{~ms}\right)$. 
boiling point of the cryogen due to strong evaporation in spray. As a result, significant cooling of the skin is achieved at the beginning of spray when cold droplets impinge onto the skin surface and boiling of cryogen is the major mode of heat transfer. As more and more droplets arrive at the skin surface, a liquid cryogen pool will be formed on the skin surface [15]. When the surface temperature reduces to below the boiling point of the cryogen, no phase change can take place, and heat has to be conducted out through the liquid layer on the surface. Since the liquid cryogen is also a poor thermal conductor, cooling becomes less efficient. The surface temperature is then slowly increased during CSC and finally stabilized at the boiling point of the cryogen $\left(-26^{\circ} \mathrm{C}\right)$.

The cooling effect of CSC with various cryogens can be examined by adjusting the parameter $h_{o, \max }$ in eq. (4) in the model. Three cryogens are examined here, cryogen R134a $\left(-26^{\circ} \mathrm{C}\right), \mathrm{R} 407 \mathrm{c}\left(-43^{\circ} \mathrm{C}\right)$, and R404a $\left(-48^{\circ} \mathrm{C}\right)$. During calculations, for a given spurt duration, the parameter $h_{o, \max }$ is varied until the final skin surface temperature approaches and is then stabilized at the corresponding boiling point of the cryogen used. Figure 4 shows the calculated variation of the skin surface temperature for three cryogens, R134a, $\mathrm{R} 407 \mathrm{c}$, and R404a. The spurt duration is $100 \mathrm{~ms}$ for all three cases. As one can see, similar cooling characteristics are predicted, as expected, for all three cryogens. The results does confirm the fact that the cryogen of a lower boiling point (e.g., R404a) will have much stronger cooling effect than the cryogen at a higher boiling point (R134a). In the case of $\mathrm{R} 404 \mathrm{a}$, the minimum surface temperature can arrive at about $-56^{\circ} \mathrm{C}$, much lower than $-32^{\circ} \mathrm{C}$ possible for R134a.

\subsection{Thermal responses of the targeted blood vessel}

In this section, we examine the thermal response of an individual targeted blood vessel obtained from our micro-model based on a Krogh-unit. We mainly consider the two morpho-

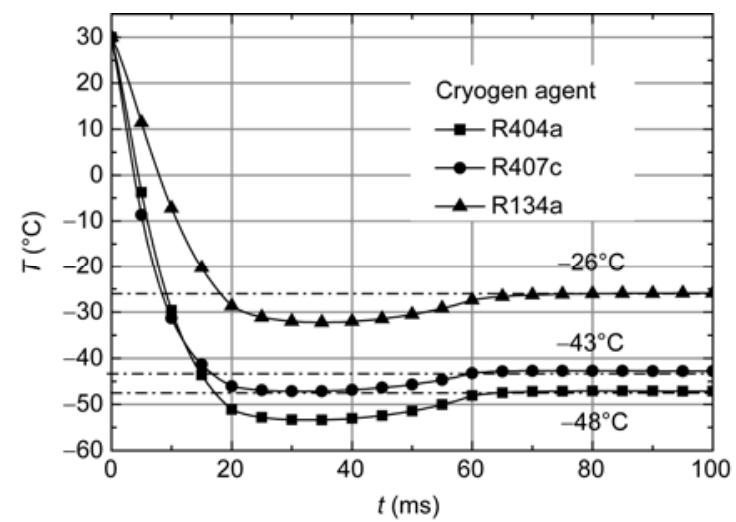

Figure 4 Dynamic variation of centerline skin surface temperature with time for three cryogens: R134a, R407c, and R404a ( $r=0, z_{t}=0$, spurt duration: $100 \mathrm{~ms}$ ). logical parameters relating to the blood vessel in PWSs, i.e., the diameter $(d)$ and the burying depth $\left(z_{t}\right)$ of the blood vessel, under various laser pulse durations $\left(t_{p}\right)$ and laser fluence $(F)$. one needs to point out that the present integrated model takes advantage of the simplicity of the classical homogeneous model for light propagation in the PWS and meanwhile the model considers the detailed geometric effect of the blood vessel through the Krogh unit, as does a discrete blood vessel model [35]. The validity of a homogeneous model to treat light propagation and distribution in complex PWSs containing dermis has been demonstrated recently through our two-temperature models $[33,37]$. The treatment of a single blood vessel in a Krogh unit is similar to the early single discrete model by Gemert et al. [38]. In those early models, the sheltering effect of the blood vessels in front of the targeted blood vessel was represented by a thick blood layer, however, while in our model the effect is more realistically represented.

(i) Thermal characteristics of a targeted blood vessel. First, we examine the general thermal characteristics of the single blood vessel buried deep in dermis under laser irradiation. Figure 5 gives the temperature distribution over the Krogh-unit at three sequential time instants, at the end of laser irradiation (a), $5 \mathrm{~ms}$ (b) and $20 \mathrm{~ms}$ (c) after a $6 \mathrm{~ms}$ pulse of laser irradiation (Note that the blood vessel locates at the center of the Krogh-unit). Such a temperature distribution and its temporal variation in Figure 5 are typical and similar to that predicted by the discrete blood vessel models $[18,38]$, demonstrating the principle of selective light absorption by the blood in the vessel. During laser irradiation, laser energy is selectively absorbed by the blood and raises the blood temperature significantly high. A large temperature difference between the blood and the surrounding dermis leads to fast heat conduction from the blood to dermal tissues, resulting in a tower-like temperature distribution over the Krogh-unit, Figure 5(a). After laser pulse was over, conduction continues, heat in the blood vessel was dissipated into the surrounding dermal tissues as one can see from Figure 5(b) and (c).

(ii) Laser pulse duration versus vessel diameter. The effect of laser pulse duration $\left(t_{p}\right)$ has widely been examined by the early researchers [39-41], leading to Anderson and Parrish' selective photothermolysis principle [2]. Figure 6 shows the results from the present model for three laser pulses with all other conditions remained the same. In this case, the shortest pulse $(1.5 \mathrm{~ms})$ clearly leads to a desired heating of the blood as shown by the rise of the blood temperature above $90^{\circ} \mathrm{C}$, while the longest pulse $(6 \mathrm{~ms})$ will not be able to raise the blood temperature high enough to cause damage of the vessel. These results are in consistent with that from the early models as well as experimental and clinic observations, demonstrating the soundness of the present model.

One important understanding from the principle of selective photothermolysis is the possible miss-match between 

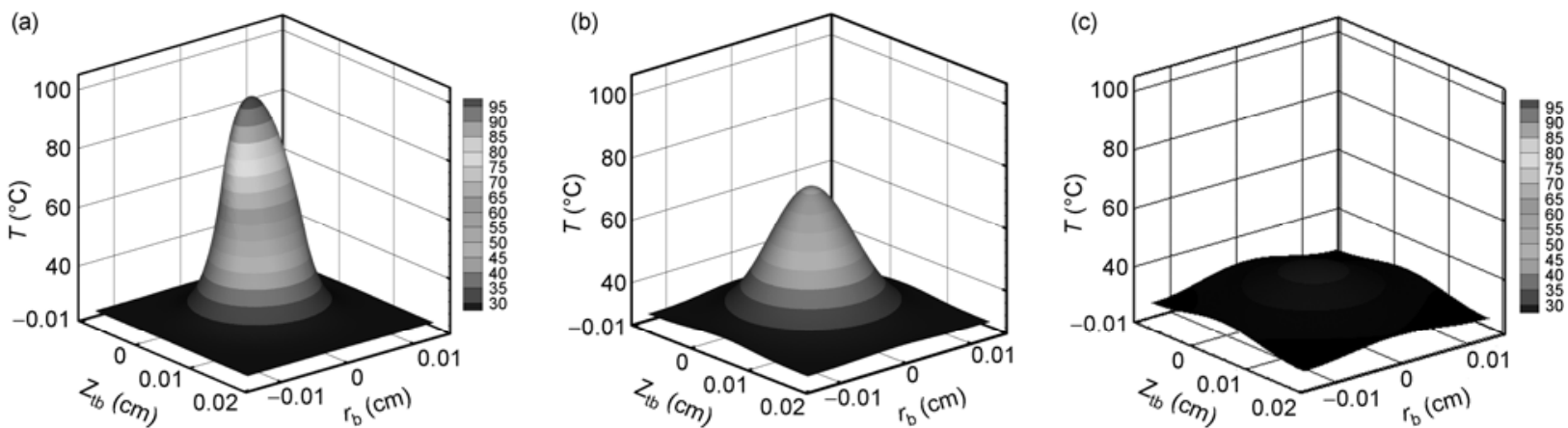

Figure 5 Temperature distribution over the Krogh-unit at three sequential time instants: (a) the end of, (b) $5 \mathrm{~ms}$ after, and (c) $20 \mathrm{~ms}$ after laser irradiation (Vessel diameter: $d=100 \mu \mathrm{m}$, bury depth: $z_{t}=200 \mu \mathrm{m}$, laser pulse duration: $t_{p}=6 \mathrm{~ms}$, laser fluence: $F=5 \mathrm{~J} / \mathrm{cm}^{2}, 100 \mathrm{~ms}$ R134a spray)
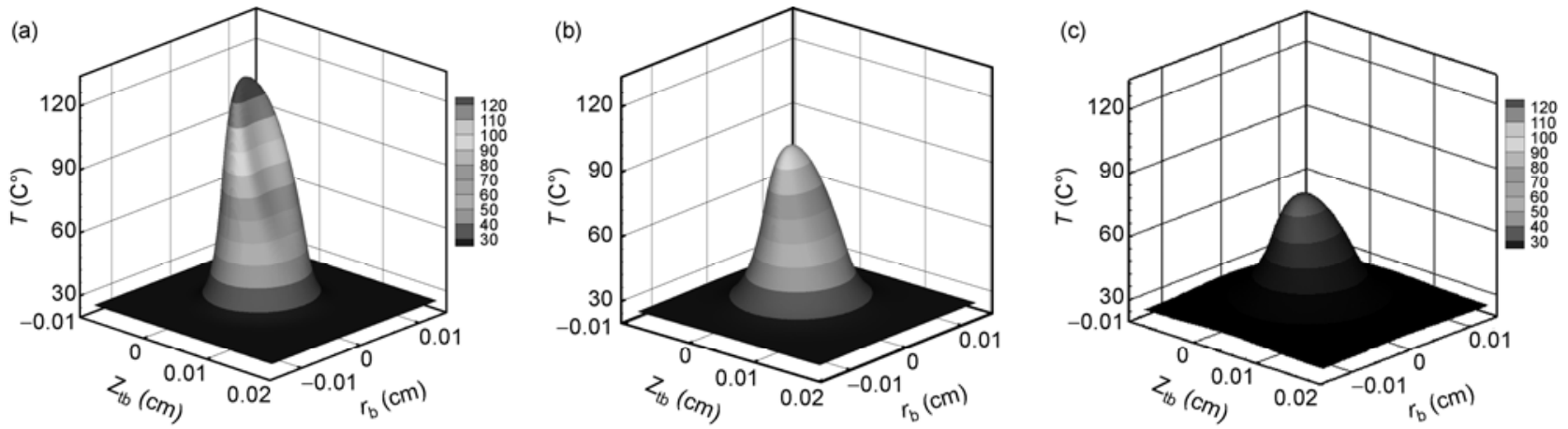

Figure 6 Effect of the pulse duration on the temperature distribution over the Krogh-unit: $t_{p}=1.5 \mathrm{~ms}(\mathrm{a}), 6 \mathrm{~ms}(\mathrm{~b})$ and $13.5 \mathrm{~ms}(\mathrm{c})\left(d=100 \mu \mathrm{m}, z_{t}=200 \mu \mathrm{m}\right.$, $F=5 \mathrm{~J} / \mathrm{cm}^{2}, 100 \mathrm{~ms} \mathrm{R} 134 \mathrm{a}$ spray).

the vessel size and the laser pulse duration. For a given size of the blood vessel, there should be an optimal laser pulse duration that can cause vessel damage. Such a phenomenon can be clearly illustrated from our results given in Figure 7 which presents the temperature distributions over the Krogh-unit at the end of laser irradiation for three vessel diameters $(d=50,100$, and $150 \mu \mathrm{m})$ under the same laser pulse duration $\left(t_{p}=6 \mathrm{~ms}\right)$ and laser fluence $\left(F=5 \mathrm{~J} / \mathrm{cm}^{2}\right)$. For this laser pulse duration, the vessel of $d=100 \mu \mathrm{m}$ should have the best heating effect. For a smaller vessel, see Figure 7(a) with $d=50 \mu \mathrm{m}$, the overall temperatures of the blood was low and significant heating occurred for dermal tissue around the vessel. On the other hand, for a larger vessel, see Figure 7(c) with $d=150 \mu \mathrm{m}$, although the highest blood temperature may raise high this time, a significant portion of the blood within the vessel (in the rear of the vessel along the path of light propagation) did not absorb enough heat due to strong attenuation of the light within the blood. Insufficient heating of the rear part of the vessel may lead to incomplete damage of the vessel, as observed in [42].

A quantitative comparison of the heating effect of different vessel diameters in Figure 7 can be made by examining the
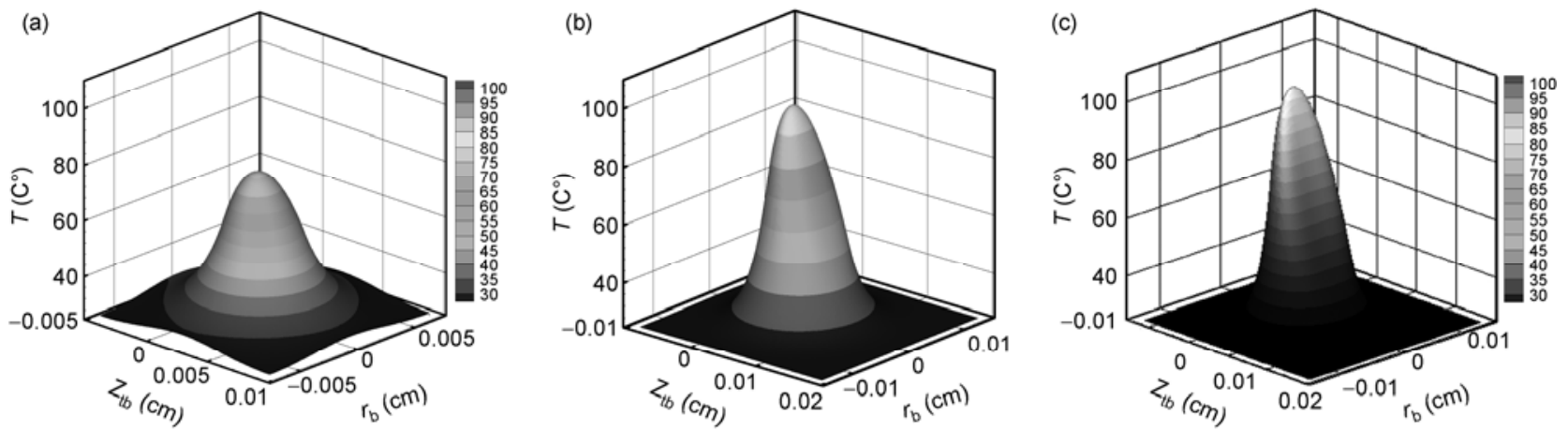

Figure 7 Temperature distributions over the Krogh-unit at the end of laser irradiation: effect of vessel diameter $d=50 \mu \mathrm{m}$ (a), $100 \mu \mathrm{m}$ (b) and $150 \mu \mathrm{m}$ (c) $\left(z_{l}=200 \mu \mathrm{m}, t_{p}=6 \mathrm{~ms}, F=5 \mathrm{~J} / \mathrm{cm}^{2}, 100 \mathrm{~ms}\right.$ R 134 a spray $)$. 
variation of the mean blood temperatures within the vessel as a function of the time during laser irradiation, as given in Figure 8 . The mean temperature over the cross-section of the blood vessel should be a better indicator for vessel damage than the highest blood temperature in the vessel. This is especially true if one considers the fact that the main goal of the laser surgery is to cause the damage of the vessel wall not the blood itself. As one can see from Figure 8, the mean temperatures in all three cases increase monotonically with time during laser pulse $\left(t_{p}=6 \mathrm{~ms}\right)$, with the fastest growth for the vessel of $d=100 \mu \mathrm{m}$ and the slowest growth for the vessel of $d=50 \mu \mathrm{m}$. At the end of $6 \mathrm{~ms}$ laser irradiation, the mean temperature for the vessel of $100 \mu \mathrm{m}$ reaches about $74^{\circ} \mathrm{C}$ while only $48^{\circ} \mathrm{C}$ for the vessel of $50 \mu \mathrm{m}$. For the vessel of $d=150 \mu \mathrm{m}$, which has the highest blood temperature of above $100^{\circ} \mathrm{C}$ (in comparison, there is only $90^{\circ} \mathrm{C}$ for $d=100 \mu \mathrm{m}$ and $70^{\circ} \mathrm{C}$ for $d=50 \mu \mathrm{m}$ ), the highest mean temperature at the end of irradiation is only $68^{\circ} \mathrm{C}$, lower than that for $d=100 \mu \mathrm{m}$. For a given laser pulse duration, there should be an optimal vessel size whose thermal relaxation time matches the pulse duration, leading to the best heating effect for the vessel. For the vessels with a smaller size, the pulse duration exceeds their thermal relaxation times and, as a result, the heat absorbed by blood would diffuse away from the vessel. For the vessels with a larger size, on the other hand, the pulse duration is less than their thermal relaxation times. There will be insufficient heat deposition within the blood vessel lumen, leading to insufficient blanching of the vessel wall. The mean temperatures from the present model calculations provide a quantitative indicator for the threshold of laser radiant needed for vessel damage for a given diameter of the targeted blood vessel.

(iii) Threshold laser fluence and effect of vessel diameter. In clinic practice, the physician needs to make sure that the blood vessels in PWS reach certain temperature to cause thermal injury of the vessel walls. It was suggested that a $100^{\circ} \mathrm{C}$ of the mean temperature of the blood within the

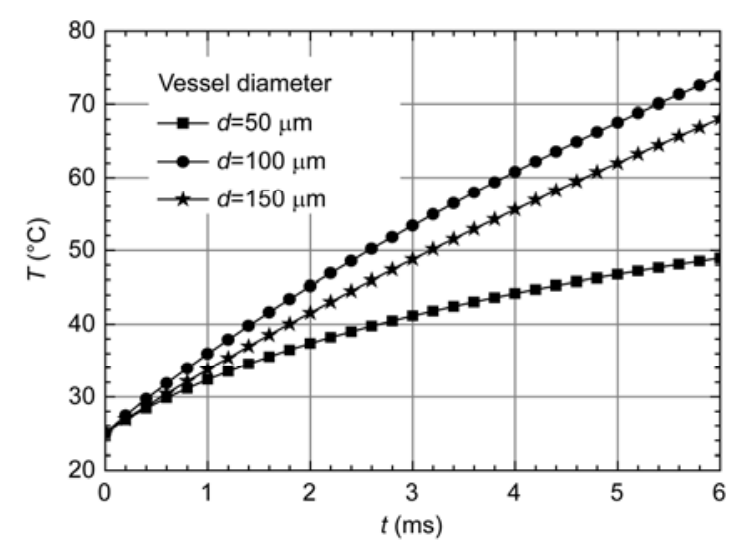

Figure 8 Variation of the mean blood temperature within the vessel during laser irradiation for three vessel sizes: $d=50,100$ and $150 \mu \mathrm{m}$, corresponding to Figure 7 ( $z_{l}=200 \mu \mathrm{m}, t_{p}=6 \mathrm{~ms}, F=5 \mathrm{~J} / \mathrm{cm}^{2}, 100 \mathrm{~ms}$ R134a spray). vessel should cause enough vessel injury [35]. Accordingly, a laser energy threshold for thermal vascular injury will be defined as the laser energy that is needed to cause the mean vessel temperature to approach $100^{\circ} \mathrm{C}$ with the laser pulse duration matches the corresponding relaxation time defined by Anderson and Parrish [2]. Typical results of such quantification are given in Figure 9 that shows the variation of the vessel mean temperature with time during laser irradiation for three sizes $(d=50,100$, and $150 \mu \mathrm{m})$ of the targeted blood vessel buried at the same depth in dermis. The corresponding laser pulses are $t_{p}=1.5,6$, and $13.5 \mathrm{~ms}$. To make sure that the final mean temperature of the vessel reaches $100^{\circ} \mathrm{C}$, the corresponding laser fluence, i.e., the laser energy threshold, would be, $F_{c}=5.5,7.7$, and $10.4 \mathrm{~J} / \mathrm{cm}^{2}$, respectively. It is interesting to notice that these numbers are fairly higher than those obtained by Lucassen et al. [35] from their single blood vessel model. This is probably because our model takes into account the scattering of the light by dermis and absorptions of light by blood vessels in front of the targeted vessel.

(iv) Effect of depth of vessel. Due to the existence of the dermis scattering and blood absorption of the light in front of the targeted blood vessel, the burying depth of the targeted blood vessel in the dermis would have a significant effect on the thermal response of the targeted vessel and the laser energy threshold for thermal vascular injury of the vessel. The effect of the burying depth on the thermal response of the targeted vessel can be illustrated by comparing the temperature distributions at the end of the laser irradiation for three locations in dermis: $z_{t}=100,200$, and 300 $\mu \mathrm{m}$, as shown in Figures 10 and 11. As expected, a stronger attenuation of laser light in the PWS-containing dermis due to scattering and absorption, leads to less energy deposition in the vessel as the vessel burying depth increases. As a result, the temperature of the blood vessel at the end of laser

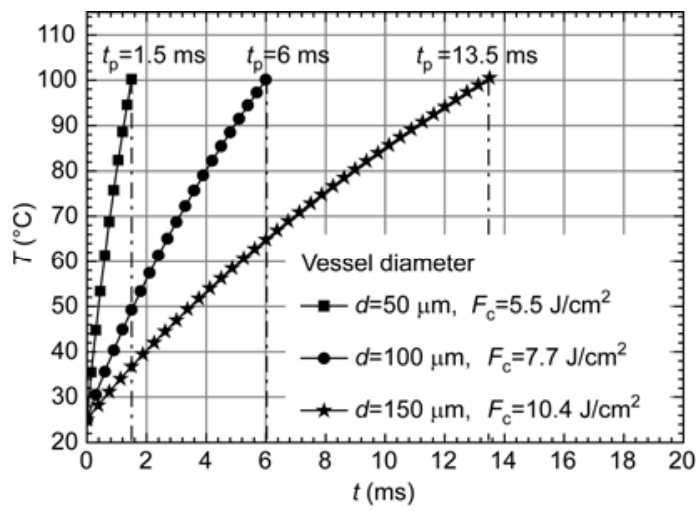

Figure 9 Variation of the mean temperature of the blood vessel during laser irradiation for three vessel sizes: $d=50,100$ and $150 \mu \mathrm{m}$. The laser pulse duration was selected, respectively, based on the corresponding relaxation time of the given vessel size: $t_{p}=1.5 \mathrm{~ms}$ for $50 \mu \mathrm{m}$ vessel, $6 \mathrm{~ms}$ for $100 \mu \mathrm{m}$ vessel, and $13.5 \mathrm{~ms}$ for $150 \mu \mathrm{m}$ vessel. The corresponding laser energy threshold $\left(F_{c}\right)$ for thermal vascular injury is $F_{c}=5.5,7.7$, and 10.4 $\mathrm{J} / \mathrm{cm}^{2}\left(z_{t}=200 \mu \mathrm{m}, 100 \mathrm{~ms}\right.$ R134a spray). 

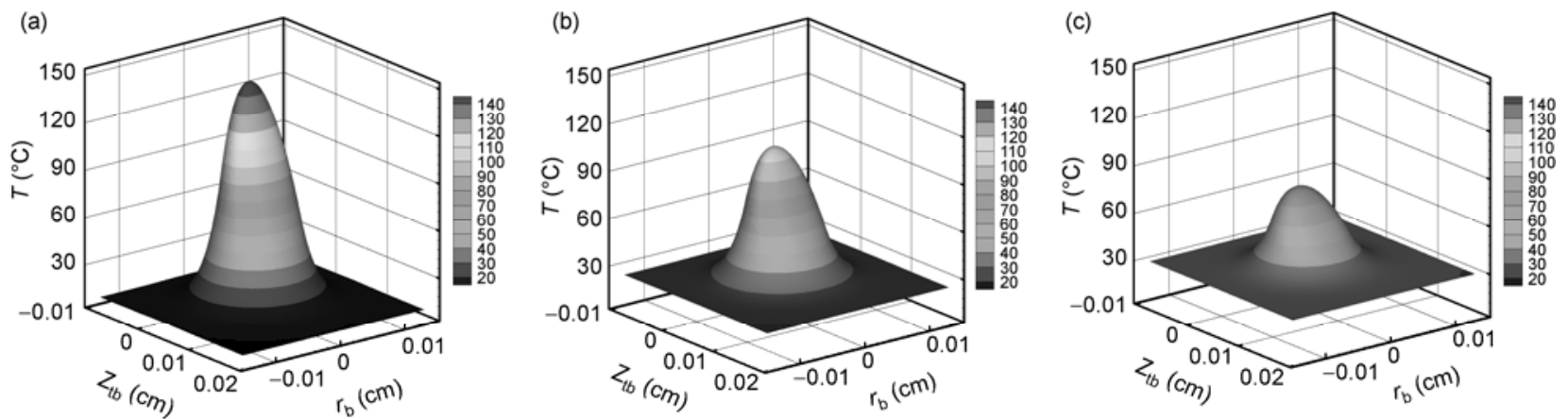

Figure 10 Temperature distributions over the Krogh-unit at the end of laser irradiation: effect of vessel depth $z_{t}=100 \mu \mathrm{m}(\mathrm{a}), 200 \mu \mathrm{m}$ (b) and $300 \mu \mathrm{m}$ (c) ( $d=100 \mu \mathrm{m}, t_{p}=6 \mathrm{~ms}, F=5.6 \mathrm{~J} / \mathrm{cm}^{2}, 100 \mathrm{~ms}$ R134a spray).

irradiation becomes lower as the vessel is buried deeper. For example, for the same conditions given in Figure 10, the mean temperature reached at the end of laser irradiation is reduced from about $100^{\circ} \mathrm{C}$ (no boiling included in the model) for $z_{l}=100 \mu \mathrm{m}$, to about $78^{\circ} \mathrm{C}$ for $z_{l}=200 \mu \mathrm{m}$, and about $61^{\circ} \mathrm{C}$ for $z_{l}=300 \mu \mathrm{m}$, see Figure 11 (Note that Figure 11 shows the mean temperature of the vessel instead of the highest temperature at the tip of the tower within the vessel as given in Figure 10). It is now recognized that the penetration depth of the short wavelength lasers like pulsed dye laser $(585 \mathrm{~nm})$ may be one of the main reasons for incomplete clearance of the PWS for most of patients, especially adult patients. Longer wavelength length such as NG:YAG laser with $1064 \mathrm{~nm}$ wavelength may be the better choice for the clearance of the deep burred PWS vessels [33].

The laser energy threshold can also be quantified by our model as done in Figure 9 for various locations of the targeted vessels in dermis. Figure 12 show the variation of the mean temperature of the vessel during laser irradiation $\left(t_{p}=6\right.$ ms) for three burying locations of Figure 11. In this case, however, the laser fluences were adjusted to the corresponding threshold fluences so the mean temperature over the vessel blood reaches $100^{\circ} \mathrm{C}$. The corresponding threshold

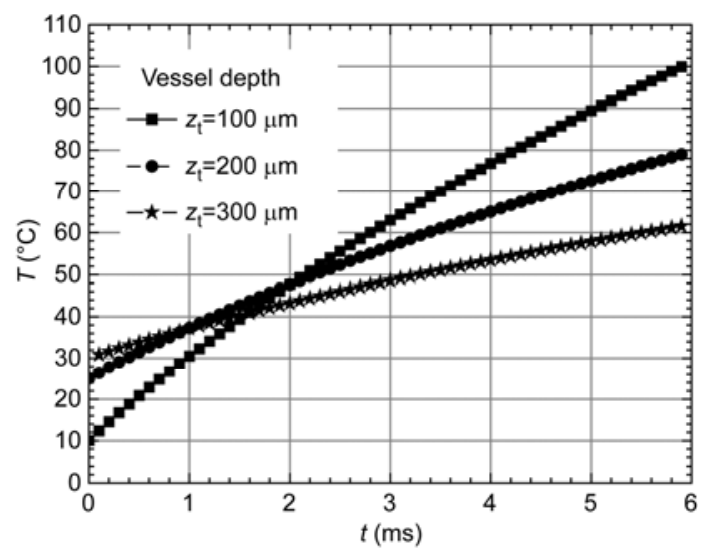

Figure 11 Variation of mean temperature of a $100 \mu \mathrm{m}$ blood vessel during laser irradiation for three tissue depths: $z_{t}=100,200$, and $300 \mu \mathrm{m}$ (all conditions are the same as in Figure 10).

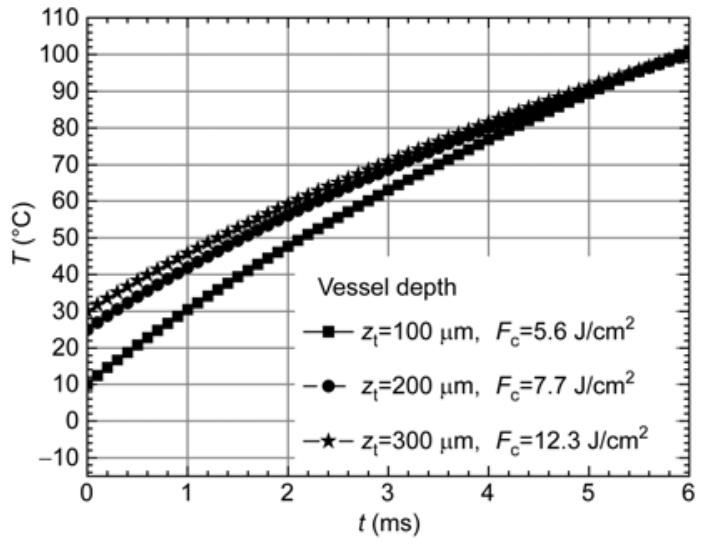

Figure 12 Variation of mean temperature of a $100 \mu \mathrm{m}$ blood vessel during laser irradiation for three tissue depths $z_{l}=100,200$, and $300 \mu \mathrm{m}$ at their corresponding laser threshold fluences: $F_{c}=5.6,7.7$, and $12.3 \mathrm{~J} / \mathrm{m}^{2}$ ( $d=100 \mu \mathrm{m}, t_{p}=6 \mathrm{~ms}, 100 \mathrm{~ms} \mathrm{R} 134 \mathrm{a}$ spray).

fluences are 5.6, 7.7, and $12.3 \mathrm{~J} / \mathrm{cm}^{2}$, respectively. As expected, higher threshold fluence is required for deeper vessel of the same size. It is also noticed in both Figures 11 and 12 that the initial mean temperature of the vessel is different for different locations. This is because the model includes the early CSC spray of R134a of 100 ms. A fairly long spray of $100 \mathrm{~ms}$ may lower down the dermis temperature at $100 \mu \mathrm{m}$ to about $10^{\circ} \mathrm{C}$ from initial $30^{\circ} \mathrm{C}$. Our macro-thermal model is able to include such cooling effect of CSC. The cooling effect on the dermis at $300 \mu \mathrm{m}$ deep is fairly small, however.

(v) Operational map of threshold laser fluence. To provide a better understanding of the thermal response of blood vessels of various sizes and burying depths in PWSs, we generate an operational map of threshold fluence $\left(F_{c}\right)$ of laser energy for thermal vascular injury over a range of the vessel diameters $(d=25$ to $150 \mu \mathrm{m})$ and the burying depths $\left(z_{t}=100\right.$ to $\left.400 \mu \mathrm{m}\right)$ as shown in Figure 13. For a given vessel diameter, the corresponding relaxation time estimated from the Anderson's model [2] is used to select the appropriate laser pulse duration. As one can see, there is basically a monotonic increase of $F_{c}$ with both $z_{t}$ and $d$. For a given 


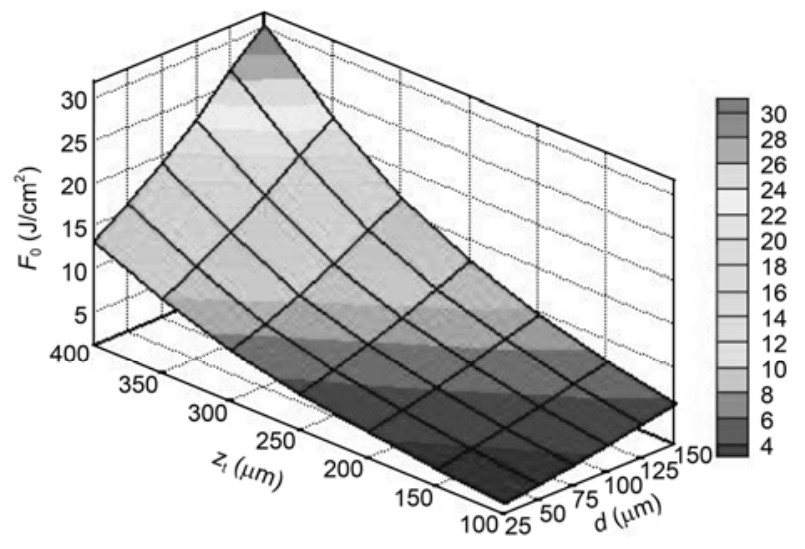

Figure 13 The threshold fluence of laser energy for thermal vascular injury as a function of the vessel depth $\left(z_{t}\right)$ and the vessel diameter $(d)$. Note that the laser pulse duration is set to be the relaxation time of the given vessel diameter (100 ms R134a Spray).

burying depth $z_{t}$, the vessel of a larger diameter requires a higher laser fluence to achieve thermal injury. For a given vessel diameter, the closer to the surface of the vessel, the less energy required to cause vessel damage. The data in Figure 13 indicate that significant high laser fluences are needed in order to achieve thermal vascular injury of large size vessels buried deep in dermis, for example, a fluence of up to $30.5 \mathrm{~J} / \mathrm{cm}^{2}$ is needed to reduce thermal injury to a vessel of $150 \mu \mathrm{m}$ buried at $400 \mu \mathrm{m}$ from the surface, even under the right laser pulse duration. Such high laser fluence, however, may create significant injury to the tissues near the surface, however. In clinic practice, significant lower laser fluences are used, for example $8-10 \mathrm{~J} / \mathrm{cm}^{2}$ [8] and, therefore, large vessels buried deeply in dermis are most likely to survive from the treatment. Efforts are now being made to address such problems [33].

\section{Conclusions}

In this paper, we present a new model for laser treatment of PWSs with cryogen spray cooling. The model can quantitatively simulate the cooling of skin during CSC and the heating of a targeted vessel in PWSs during laser irradiation, with an accurate consideration of the sheltering effect of blood vessels and dermal tissues. The effect of cryogen type and spurt duration on skin cooling was examined. The results indicate that the cryogen of a lower boiling point such as R404a may provide better protection of the skin. The new model can be used to conduct thermal analysis of a targeted vessel in PWSs, based on a microscopic Krogh-unit. The model introduces two morphological parameters of the targeted vessel, the diameter and the burying depth. A detail analysis with the model is conducted to examine the effect of such two parameters on thermal response of the targeted vessels under various clinic conditions. The threshold laser fluence for thermal vascular injury was estimated for given vessel diameter and depth. An operational map is generated for threshold fluences over a wide range of vessel diameters and vessel depths. The results can be used to explain the origin of the difficulty to achieve complete clearance of the PWSs by the pulsed dye lasers, and to provide guidance for optimal setting of the clinic parameters in laser treatment of PWSs.

The work was supported by Joint Research Fund for Cverseas Chinese Scholars (51228602), the Key Project of National Natural Science Foundation of China (51136004) and the Ph.D Programs Foundation of Ministry of Education of China (20110201110038).

1 Barsky S H, Rosen S, Geer D E, et al. The nature and evolution of port wine stains: A computer-assisted study. J Invest Dermatol, 1980, 74: $154-157$

2 Anderson R R, Parrish J A. Microvasculature can be selectively damaged using dye laser: A basic theory and experimental evidence in human skin. Lasers Surg Med, 1981, 1: 263-276

3 Tunnell J W, Nelson J S, Torres J H, et al. Epidermal protection with cryogen spray cooling during high fluence pulsed dye laser irradiation: An ex vivo study. Lasers Surg Med, 2000, 27: 373-383

4 Aguilar G, Majaron B, Verkruysse W, et al. Theoretical and experimental analysis of droplet diameter, temperature, and evaporation rate evolution in cryogenic sprays. Int J Heat Mass Transfer, 2001, 44: 3201-3211

5 Aguilar G, Verkruysse W, Majaron B, et al. Measurement of heat flux and heat transfer coefficient during continuous cryogen spray cooling for laser dermatologic surgery. IEEE J Sel Top Quant, 2001, 7: 1013-1021

6 Nelson J S, Milner T E, Anvari B, et al. Dynamic epidermal cooling during pulsed laser treatment of port-wine stain: A new methodology with preliminary clinical evaluation. Arch Dermatol, 1995, 6: 695700

7 Fiskerstrand E J, Svaasand L O, Kopstad G, et al. Laser treatment of port wine stains: Therapeutic outcome in relation to morphological parameters. Brit J Dermatol, 1996, 134: 1039-1043

8 Bernstein E F. Treatment of a resistant port-wine stain with a new variable pulse-duration pulsed-dye laser. J Cosmet Dermatol, 2008, 7: 139-142

9 Fiskerstrand E J, Svaasand L O, Kopstad F, et al. Photothermally induced vessel-wall necrosis after pulsed dye laser treatment: Lack of response in port-wine stains with small size of deeply located vessels. J Invest Dermatol, 1996, 107: 671-675

10 Li D, He Y L, Wang G X. Thermal Modelling for Laser Treatment of Port Wine Stains. In: Bernardes M A S, ed. Developments in Heat Transfer. Croatia: InTech Publishers, 2011. 537-556

11 Aguilar G, Diaz S H, Lavernia E J, et al. Cryogen spray cooling efficiency: Improvement of port wine stain laser therapy through multiple-intermittent cryogen spurts and laser pulses. Lasers Surg Med, 2002, 31: 27-35

12 Pfefer T J, Smithies D J, Milner T E, et al. Bioheat transfer analysis of cryogen spray cooling during laser treatment of Port Wine Stains. Lasers Surg Med, 2000, 26: 145-157

13 Majaron B, Verkruysse W, Kelly K M, et al. Er:YAG laser skin resurfacing using repetitive long-pulse exposure and cryogen spray cooling: II. Theoretical analysis. Lasers Surg Med, 2001, 28: 131-137

14 Verkruysse W, Majaron B, Tanenbaum B S, et al. Optimal cryogen spray cooling parameters for pulsed laser treatment of port wine stains. Lasers Surg Med, 2000, 27: 165-170

15 Aguilar G, Wang G X, Nelson J S. Effect of spurt duration on the heat transfer dynamics during cryogen spray cooling. Phys Med Biol, 2003, 48: 2169-2181

16 Franco W, Liu J, Wang G X, et al. Radial and temporal variations in surface heat transfer during cryogen spray cooling. Phys Med Biol, 
2005, 50: 387-397

17 Aguilar G, Diaz S H, Lavernia E J, et al. Effect of time-dependent boundary conditions on epidermal tissue damage during Port Wine Stain laser surgery. In: Proceedings of the International Mechanical Engineering Congress and Exposition (IMECE). New York: ASME Press, 2001, 52: 43-48

18 Jia W C, Choi B, Franco W, et al. Treatment of cutaneous vascular lesions using multiple-intermittent cryogen spurts and two-wavelength laser pulses: Numerical and animal studies. Lasers Surg Med, 2007, 39: 494-503

19 Li D, He Y L, Liu Y W, et al. Numerical analysis of cryogen spray cooling of skin in dermatologic laser surgery using realistic boundary conditions. In: 22th International Congress Refrigeration Conference. Beijing. 2007

20 Li D, He Y L, Wang G X, et al. Numerical analysis of cold injury of skin in cryogen spray cooling for dermatologic laser surgery. In: Proceedings of the International Mechanical Engineering Congress and Exposition (IMECE). Seattle: ASME Press, 2007, 8: 673-681

21 Kerjzer M, Jacques S L, Prahl S A, et al. Light distributions in artery tissue: Monte-Carlo simulations for finite diameter laser beams. Lasers Surg Med, 1989, 9: 148-154

22 Lucassen G W, Verkruysse W, Keijzer M, et al. Light distribution in a port wine stain model containing multiple cylindrical and curved blood vessels. Lasers Surg Med, 1996, 18: 345-357

23 Pickering J W, van Gemert M J C. $585 \mathrm{~nm}$ for the laser treatment of port-wine stains: A possible mechanism. Lasers Surg Med, 1991, 11: 616-618

24 Kienle A, Hibst R. A new optical wavelength for treatment of port wine stains? Phys Med Biol, 1995, 40: 1559-1576

25 Verkruysse W, Pickering J W, Beek J F, et al. Modeling the effect of wavelength on the pulsed dye laser treatment of port wine stains. Appl Opt, 1993, 32: 393-398

26 Krogh A. The number and distribution of capillaries in muscles with calculations of the oxygen pressure head necessary for supplying the tissue. J Physiol, 1919, 6: 409-415

27 Barsky S H, Rosen S, Geer D E, et al. The nature and evolution of port wine stains: A computer-assisted study. J Invest Dermatol, 1980, 74: $154-157$

28 Wang L H, Jacques S L, Zheng L Q. MCML-Monte Carlo modeling of light transport in multi-layered tissues. Comput Meth Prog Biol, 1995, 47: 131-146

29 Welch A J, Wissler E H, Priebe L A. Significance of blood flow in calculation of temperature in laser irradiated tissue. IEEE Trans Biomed Eng, 1980, BME-27: 164-166

30 Wissler $\mathrm{E} \mathrm{H}$. An analysis of chorioretinal thermal response to intense light exposure. IEEE Trans Biomed Eng, 1980, BME-23: 209-215

31 Zhou Z F, Wang G X, Guo L J, et al. Comparison and evaluation of evaporation models for single moving droplet with a high evaporation rate. Powder Technol, 2012, doi: 10.1016/j.powtec.2012.07.002

32 Zhou Z F, Chen B, Wang Y S, et al. An experimental study on pulsed spray cooling with refrigerant R-404a in laser surgery. Appl Therm Eng, 2011, 39: 29-36

33 Li D. Numerical and experimental studies on laser induced selective photothermolysis in biological tissue (in Chinese). Doctoral Dissertation. Xi'an: Xi' an Jiaotong University, 2011

34 Sun F, Aguilar G, Kelly K M, et al. Thermal analysis for cryosurgery of nodular basal cell carcinoma. In: Proceedings of the International Mechanical Engineering Congress and Exposition (IMECE). Chicago: ASME Press, 2006, 8: 125-131

35 Lucassen G W, Svaasand L O, Verkruysse W, et al. Laser energy threshold for thermal vascular injury in a port wine stain skin model. Laser Med Sci, 1995, 10: 231-234

36 Anvari B, Milner T E, Tanenbaum B S, et al. Selective cooling of biological tissues: Application for thermally mediated therapeutic procedures. Phys Med Biol, 1995, 40: 241-252

37 Li D, He Y L, Wang G X, et al. Numerical simulation of cryogen spray cooling during the laser treatment of the Port Wine Stain (in Chinese). J Eng Thermophys, 2008, 29: 2107-2110

38 Van Gemert M J C, Welch A J, Pickering J W, et al. Wavelengths for laser treatment of port wine stains and telangiectasia. Lasers Surg Med, 1995, 16: 147-155

39 Smithies D J, Butler P H, Day W A, et al. The effect of the illumination time when treating port-wine stains. Lasers Surg Med, 1995, 10: 93-104

40 Vangemert M J C, Henning J P H. A model approach to laser coagulation of dermal vascular lesions. Arch Dermatol Res, 1981, 270: 429-439

41 Zhou J H, Liu J. Numerical study on 3-D light and heat transport in biological tissues embedded with large blood vessels during laserinduced thermotherapy. Nnmer Heat Tr A-Appl, 2004, 45: 415-449

42 Babilas P, Shafirstein G, Baumler W, et al. Selective photothermolysis of blood vessels following flashlamp-pumped pulsed dye laser irradiation: In vivo results and mathematical modeling are in agreement. J Invest Dermatol, 2005, 125: 343-352

Open Access This article is distributed under the terms of the Creative Commons Attribution License which permits any use, distribution, and reproduction in any medium, provided the original author(s) and source are credited. 